\title{
Integrated buck and boost converter for a universal battery charger of an Electric Vehicle
}

\author{
Kondreddy Sreekanth Reddy ${ }^{*}$, Sreenivasappa B. Veeranna ${ }^{2}$ \\ ${ }^{1}$ Center for Research in Power Electronics, Presidency University, Bengaluru, Karnataka, India. \\ ${ }^{2}$ Center for Research in Power Electronics, Presidency University, Bengaluru, Karnataka, India.
}

\begin{abstract}
A two-stage converter connects the input grid voltage to a pack of batteries with the voltage varying between $48-400 \mathrm{~V}$, depending on the size and the range of the vehicle, with battery-operated electric and Plug-in Hybrid Electric Vehicles (PHEVs). This article offers a unique built-in converter that can interface with both high voltage (HV) and low voltage (LV) batteries. For all car architectures a universal charger that can accommodate this wide range of battery pack voltages is suitable. The novel integrated buck and boost converter (IBBC) is the proposed converter supplied using AC-DC driver at the front end mode. The main objective of this paper is to show a universal battery charger for an EV with a high power factor (PFC) and a small total harmonic distortion (THD) in addition to the high power density. A PFC converter is formed without any auxiliary circuit to balance the output voltage dependence of the battery against fluctuations in the ac grid input voltage, which in turn reduces the cost of additional circuit. A closed loop controller scheme is used to adjust for variations in the broad range output voltage and load. The proposed topology's detailed operation is simulated using the MATLAB/Simulink software and achieved a THD of $1.18 \%$.
\end{abstract}

\section{Introduction}

The rising popularity of battery electric vehicles (BEVs) and plug-in hybrid electric vehicles (PHEVs), also known as electric vehicles (EVs), can be attributed to a number of significant advantages, including reduced global greenhouse gas (GHG) emissions [1] and superior vehicular performance [2], as well as progressing toward cost competitiveness with internal combustion engine (ICE) vehicles in the long run. A charger is an essential component of an EV's system. In [3]-[4] EV chargers are classified as off-board chargers and on-board chargers. Customers prefer on-board chargers because they can charge their EVs anywhere. Electric vehicles (EVs) commonly charge their batteries using an on-board charger set up with power electronics arrangement. Low- and highfrequency harmonic compliance is always a concern when designing power electronic systems that are connected to a mains supply. The development of a PFC for main power equipment often aims for high efficiency and high specific power at the very same moment, with the duty cycle as a parameter. The EV on-board charger can be connected to the grid supply by an ac-dc conversion at front side, the dc link control and power control is carried out on the grid [5][7].A galvanic isolation is provided to avoid harmful issues while charging the battery with the help of dc-dc converter at the back end which connects the input grid and battery charger of EV [8]-[10]. Boost converters, with or without interleaving, are preferred in most PFC applications to provide dc output voltages greater than the peak ac line voltage [11], [12]. An increase in switch stress, a bulky inductor and a large input current ripple are some of the drawbacks of a boost converter when operating at low AC line voltages [12]. To increase power density, the effects of interleaving various parallel boost cells are proposed in [13] and compared with the conventional boost PFC topology. It is possible to achieve a universal charger by increasing or decreasing the PFC output voltage, which does not have a significant impact on magnetics of high frequency transformers. Buck-boost, cuk and SEPIC converters can meet the requirements for a universal charger that operates at worldwide voltage ranges, but they have the disadvantages of stress and bulky size. There are two ways to do this: [14], [15]. For a low stress level in the device, [14] propose combining buck and boost with two switches between the source and the load, two switch converters provide a direct energy transfer path, which reduces the effect of passive components. In this paper, we will not investigate alternative topologies, such as the bridgeless PFC circuit [11], which has been reported to have a slightly higher efficiency potential but also increased common mode (CM) noise issues. In the literature review [16]-[20], converters which are used for high voltage battery charging purpose and low voltage battery charging purpose are proposed independently and some of the authors are proposed together with the usage of more number of switches or components in the circuits and with more ripple content in the supply current which says poor power factor and the attainment of universal voltages were not showed

\footnotetext{
* Corresponding author: ksreekanthreddy@presidencyuniversty.in
} 
clearly. To overcome these challenges / drawbacks, in this paper a modified integrated Buck and Boost Converter for a Universal Battery Charger of an EV is proposed. In this the buck converter is cascaded with two boost converters connected in parallel which in turn is interleaved boost converter through which the EV batteries can be charged to either high voltage or low voltage with improved THD, power factor and the usage of components are less compared with the existing models which are discussed in the literature. The circuit along with waveform will help us understand the working of the model and how it solves the above-mentioned drawbacks.

The structure of the paper as follows: in section II the operation of the modified integrated Buck and Boost Converter for a Universal Battery Charger is discussed. The controlling scheme of current and voltage loops are discussed in section III. In section IV the results and discussions are provided and the conclusions are given in section $\mathrm{V}$.

\section{Operation of the Proposed Converter}

The circuit diagram of the proposed model is shown in fig. Which consists of power factor correction circuit with interleaved switches and a DC-DC converter for a variable dc output voltage to charge a universal battery. The circuit consists of three switches S1, S2 and S3, three inductors L1, L2 and L3 to limit the ripple currents, two capacitors C1 and $\mathrm{C} 2$ connected at the rectifier and after the DC-DC converter to maintain constant dc voltage. The combination of interleaved switches with two inductors at the input side of DC-DC converter reduces the ripple content in the input current. The switches will be turned on and off in order to attain a low dc voltage and high dc voltage at the DC-DC converter stage to charge the corresponding battery (48$400 \mathrm{~V})$ keeping the input supply as same. The gating signals are generated by two controlling schemes current and voltage feedbacks to improve the power factor while providing a wide range of output voltages. Based on the operating mode, an LC filter is balancing either at the converter's input or output. This approach eliminates the pulsating currents to the source and load.

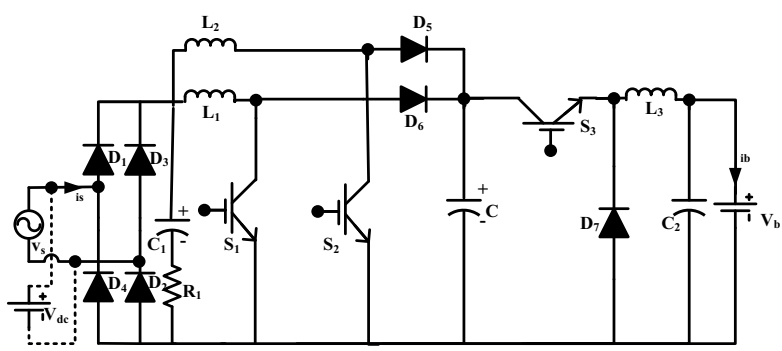

Fig. 1. Proposed modified integrated Buck and Boost Converter

The proposed circuit runs in continuous conduction way of boost and buck model based on the requirement of universal battery voltage range. If it is required to charge a high voltage battery $V_{b}$ that is greater than the rectified output voltage $V_{O R}$ or the peak value of the input supply, then the converter is operated in boost mode. The switches $S_{1}$ and $S_{2}$ are turned on and off using PWM pulses with a phase difference of 180 degrees between each switch and S3 is turned on continuously, after they are added together, the ripple current at PFC output is reduced as a result of the phase shift of PWM signals. Across the capacitor $\mathrm{C}$, the average voltage is zero and which supplies energy before the switches are on. A pair of inductors $L_{1}$ and $L_{2}$ are used to divide the input current in any of the operating mode.

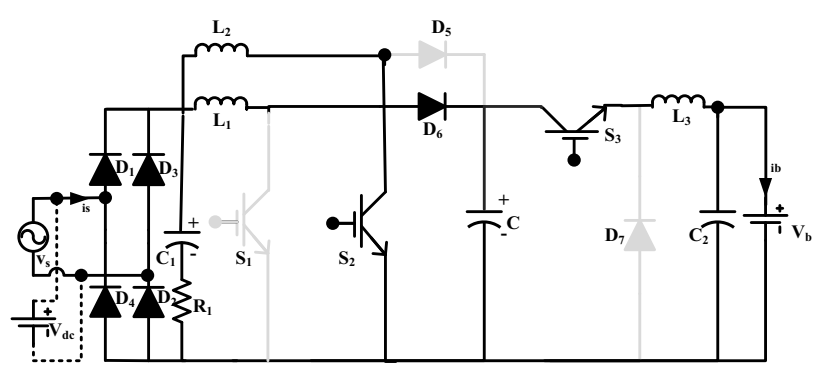

Fig. 2. Equivalent mode 1 of boost operation

When any of the first two switches $S_{1}$ or $S_{2}$ is in on position as shown in fig. 3 and fig. 2 that corresponding inductor $\mathrm{L}_{2}$ stores energy through that switch and the load or battery gets energy from the rectified output voltage $\mathrm{V}_{\mathrm{OR}}$ and the energy stored in the other inductor $\mathrm{L}_{1}$. At some point of time the two switches are turned on and off because of the phase difference then the capacitor $\mathrm{C}$ supplies energy to the battery and the two inductors supplies energy to the battery which is given in fig.4. In this way the converter operates in boost mode as the switch S3 is always on in any of those equivalent modes of boost operation, so continuity exists between the battery and interleaved converter.

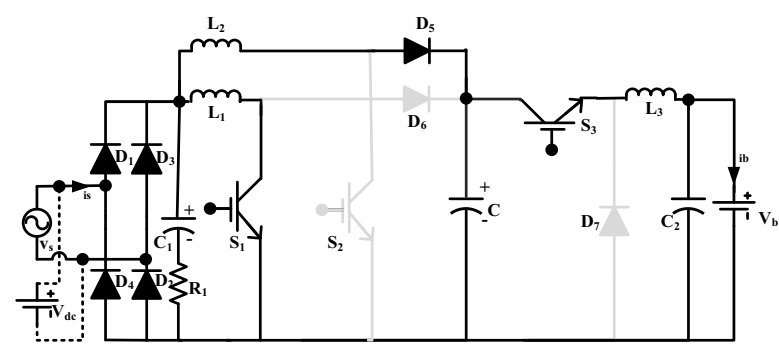

Fig. 3. Equivalent mode 2 of boost operation

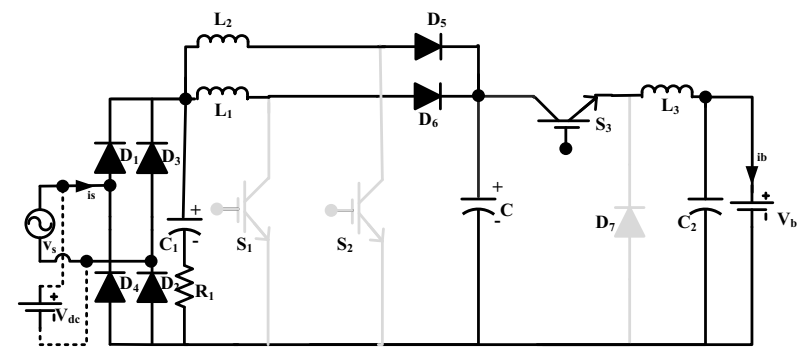

Fig. 4. Equivalent mode 1 of buck operation 


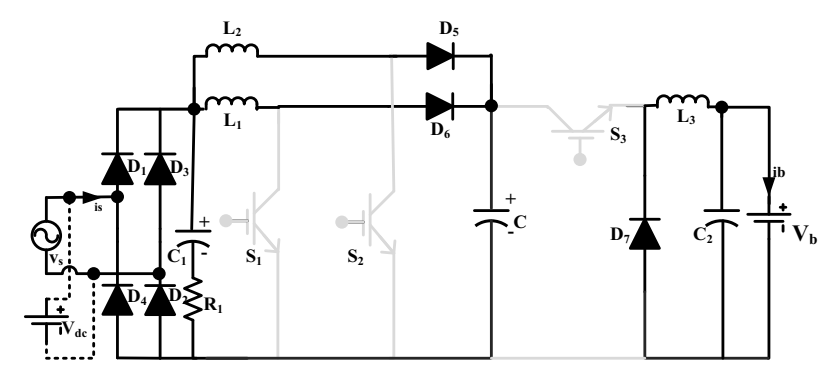

Fig. 5. Equivalent mode 2 of buck operation

The corresponding switch gating signals and the inductors current waveform are shown in fig. 6 for one mode of interleaved boost operation and the other mode can be drawn with a phase shift of 180 degrees. As switch S3 is always on in this mode and if the duty ratio is denoted by $\mathrm{d} 3=1$ and the switches $\mathrm{S} 1$ and $\mathrm{S} 2$ duty ratios are $\mathrm{d} 1$ and $\mathrm{d} 2$ then the overall $\mathrm{dc}$ gain conversion is $A=d 3 / 1-d 1$.

If the required output voltage or battery voltage is less than the rectified output voltage $\mathrm{V}_{\mathrm{OR}}$ or the peak value of the input supply then the switches $S_{1}$ and $S_{2}$ are turned off continuously and the switch $S_{3}$ is operated in PWM mode as shown in the model waveform fig. The corresponding equivalent circuit mode operation is shown in fig. 4 when the switch $S_{3}$ is in on position and in fig.5 when the switch is in off condition. When the switch is in off condition. In this way the switch $S_{3}$ gets connected to battery when it is on and when it is off the battery or load gets connected with didoe $D_{7}$ and inductor $L_{3}$. By controlling the gating signal of $S_{3}$ the buck converter operation can be achieved. The corresponding mode gating signals and the inductor current are shown in fig.7.

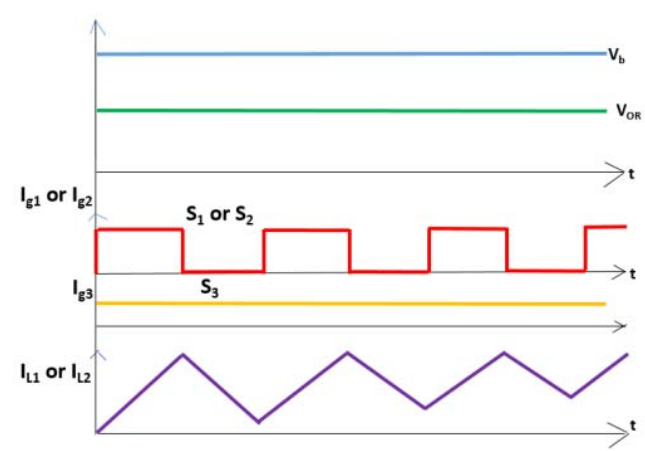

Fig.6. Model waveforms in boost operating mode

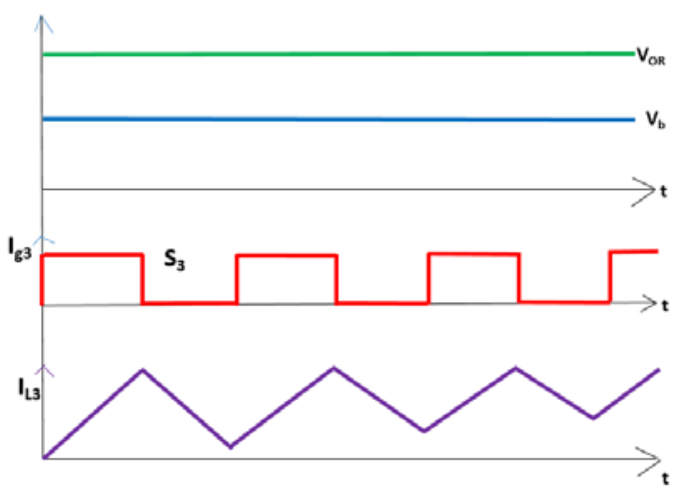

Fig.7. Model waveform in buck operating mode inductor current does not fall completely to zero in regular intervals of time. After some interval of time the current through inductors gets some minimum and maximum values from there onwards it maintains that limit. The corresponding switching states of the converter based on the requirement of battery voltage and associated mode of operation are listed in table 1.

Table 1. Switching States of the proposed converter

\begin{tabular}{lcllllll}
\hline Condition & & & S3 & D5 & D6 & D7 & Mode \\
& S1 & S2 & & & & & \\
\hline$V_{\mathbf{b}}>V_{\text {OR }}$ & n & off & on & on & off & off & Boost \\
\hline $\mathbf{V}_{\mathbf{b}}>V_{\text {OR }}$ & off & on & on & off & on & off & Boost \\
\hline$V_{\mathbf{b}}>V_{\text {OR }}$ & off & off & on & on & on & off & Boost \\
\hline$V_{\mathbf{b}}<V_{\text {OR }}$ & off & off & on & on & on & off & Buck \\
\hline$V_{\mathbf{b}}<V_{\text {OR }}$ & off & off & off & on & on & on & Buck \\
\hline
\end{tabular}

\section{Designing Parameters and Control Techniques:}

\section{i. Selection of Inductors and Capacitors:}

Under assumption of continuous conduction mode, the inductors $\mathrm{L}_{1}$ and $\mathrm{L}_{2}$ are selected with minimal effect on current ripple which is an important parameter for PFC. The input current $I_{\text {in }}$ is sum of the two inductor peak average currents $I_{L 1}$ and $I_{L 2}$.

$I_{\text {in }}=I_{L 1}+I_{L 2}$

The ripple in inducor currents are $\Delta I_{L 1}$ and $\Delta I_{L 2}$ whose magnitudes are less than the averahe inductor currents.

$$
\begin{aligned}
& \Delta I_{L 1}+\Delta I_{L 2}<I_{i n} \\
& \Delta I_{L 1}=\frac{T_{S} * V_{O R} * d_{1}}{2 L_{1}} \\
& \Delta I_{L 2}=\frac{T_{S} * V_{O R} * d_{2}}{2 L_{2}}
\end{aligned}
$$

The PFC inductor equations for continuous conduction mode are given as

$L_{1}=L_{2}=\frac{V_{C} * d_{1} *\left(1-d_{1}\right)}{2 * f_{S}}$

Where $V_{C}$ is the voltage across the the dc link capacitor $\mathrm{C}$

$f_{S}$ is the switching frequency

The duty ratio $d_{1}$ or $d_{2}$ in this interleaved boost mode are given by

$d_{l}=\frac{V_{C}-V_{O R}}{V_{O R}}$

The buck converter inductor $L_{3}$ equation for continuous conduction mode is given by

$L_{3}=\frac{V_{C}-v_{b}}{\Delta I_{L} * 2 * f_{S}} * d_{3}$

$C_{l}$ is located at the output of the bridge rectifier, $C$ is located at the high DC-link, and $C_{2}$ is located across 
the battery in the converter. These capacitors are designed using the following equations:

$$
\begin{aligned}
& C_{1}=\frac{1}{4 \sqrt{3} f R_{b} \gamma} \\
& C_{2}=\frac{V_{O R}}{4 f_{S} R \Delta V_{O R}} \\
& C_{3}=\frac{V_{b}}{4 f_{s} R_{b} \Delta V_{b}}
\end{aligned}
$$

\section{ii. Control Techniques}

To obtain a dc variable voltage and to charge a universal battery, the proposed topology's control technique is depicted in Figure 8 and 9. The implementation of proposed modified integrated buck and boost converter operation modes are required to be controlled in closed loop control model in order to get a wide output voltage range and to improve the power quality. Control technique inputs for the proposed topology is selected based on the external feedback signals taken from dc link battery voltage and PFC inductor current. The control technique used for PFC converter and dc-dc converter are explained briefly in the following sub-sections.

The control technique used for the PFC converter as shown in Fig. 8 consists of PID controller, rectifier, adder/subtractor and a comparator. The AC grid voltage is attenuated with the help of a transformer and a resistive network to obtain the required $\mathrm{AC}$ voltage. This $\mathrm{AC}$ voltage is converted to equivalent reference $\mathrm{DC}$ voltage $V_{r}$ using a rectifier and a filter. The reference battery voltage $V_{\text {bref }}$ is compared with the measured battery voltage $V_{b}$ to get the error signal. This error signal is fed to the input of PI controller to generate reference current $I_{r}$ which is compared with the measured PFC inductor current $I_{L}$ to generate controlled step signal. The step signal is compared with the high frequency saw tooth signal to obtain pulse width modulated (PWM) signal to drive the switches in PWM mode.

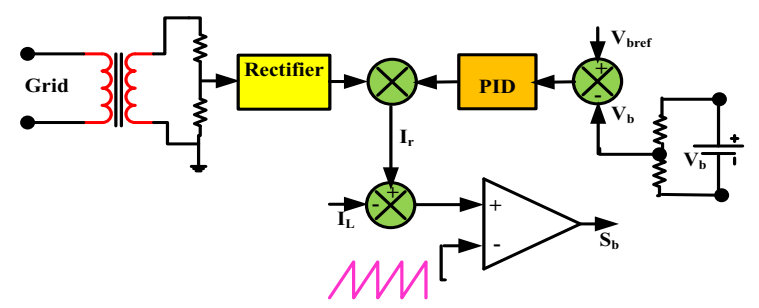

Fig. 8. Controller Scheme for PFC

The simulated model of the proposed converter along with the controller schemes are shown in fig.9 It consists of four PI controller blocks, in which two are used for boost converter operation and two are used for buck converter operation with PFC. In this the logic controller is selected based on the feedback voltage. If the actual battery voltage which is reference voltage is less than the rectified voltage then the above two controllers used to generate the gating signals to the switches $S_{1}$ and $S_{2}$ and the through the two controllers at lower side will provide a continuous gating signal to $\mathrm{S}_{3}$ switch and if reference voltage that is actual battery voltage is lower than the rectified voltage then mode selector will provide a continuous off signal to the switches $S_{1}$ and $S_{2}$ through the top two controllers and a PWM signal is generated with the help of two controllers which are at lower side and the same is provided to switch $\mathrm{S}_{3}$.

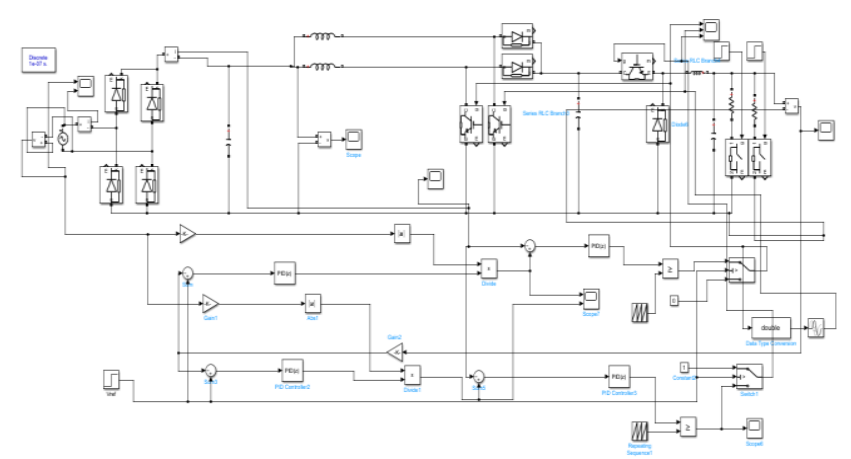

Fig. 9. Simulated model of proposed converter with controlling scheme

In this controller scheme, the reference is nothing but the rectified voltage, based on this voltage the converter is operated in boost or in buck to charge a universal battery with minimum harmonic distortion in input current.

\section{Results and Discussion}

This section provides a brief overview of the proposed topology's results simulated in MATLAB 2021a and discussions. Starting at $0.0 \mathrm{~s}$, stopping at $1 \mathrm{~s}$, and using a fixed step solver with a step size of $5 \mathrm{e}-6 \mathrm{~s}$, the model configuration parameters were selected and the battery rating is $1 \mathrm{~kW}$ and the values of inductors and capacitors are calculated as per the designing parameters with a switching frequency of $30 \mathrm{kHz}$. The Fig. 10 shows the Simulation waveforms of IBBC PFC converter operated in buck mode. The input supply voltage in red colour and the input supply current is in blue colour and the battery reference voltage is taken as $150 \mathrm{~V}$ which is projected from the simulation waveform. This shows that the supply voltage and supply currents are in phase with each other hence the power quality is improved in the buck mode. The fig. 11 and 12 shows the output waveform of battery voltage and the input grid voltage and currents. Even from the waveforms it is clear that battery voltage is $300 \mathrm{~V}$ in fig 11 and $400 \mathrm{~V}$ in fig. 12 . In fig 12 the grid voltage is scaled to $23 \mathrm{~V}$ in blue colour and current in red colour to shows the the power quality improvement. In this mode the input grid voltage of peak value is $220 \mathrm{~V}$ which means now the converter is operating in boost mode and the input voltage and currents are in phase even if it is operated in boost mode. The Interleaved Boost cascaded by Buck converter has good power quality. The different values of the output voltage can be set manually as a reference input to the 
PID controller, it means the battery voltage rating (48 to $400 \mathrm{~V}$ ) can be considered as a reference input to the PID controller in pragmatic scenario.
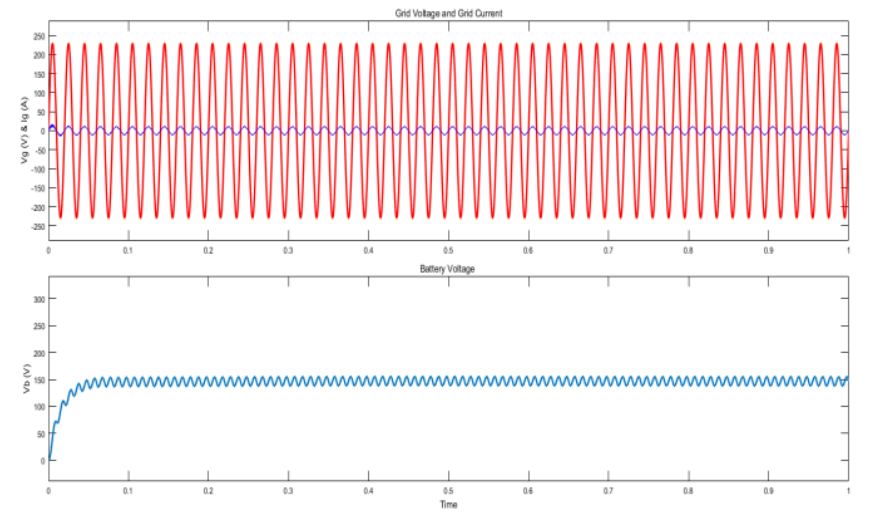

Fig. 10. Waveforms of input grid voltage, grid current and battery voltage in Buck mode
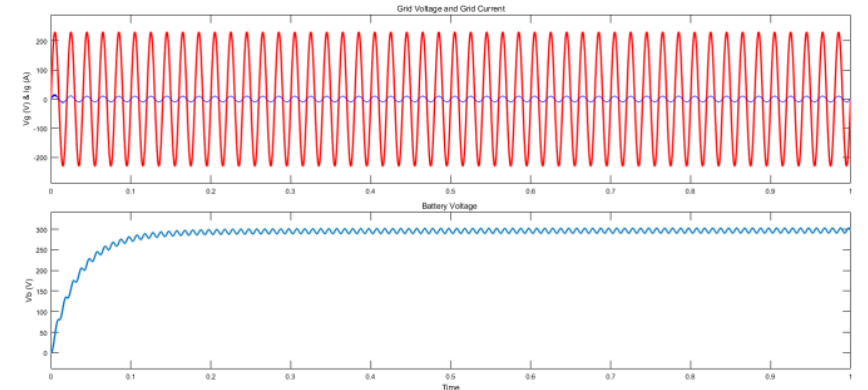

Fig. 11. Waveforms of input grid voltage, grid current and battery voltage in Boost mode (300V battery)
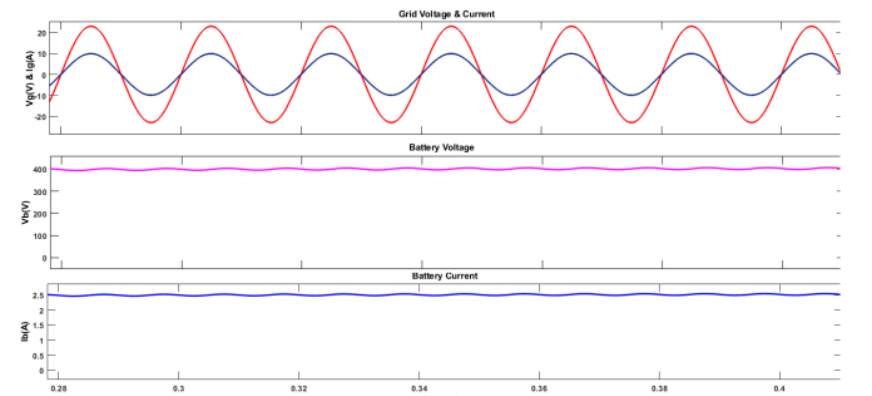

Fig. 12. Waveforms of input grid voltage, grid current and battery voltage in Boost mode (400V battery)

Through this concept, different voltage range of batteries can be charged using variable battery charger.

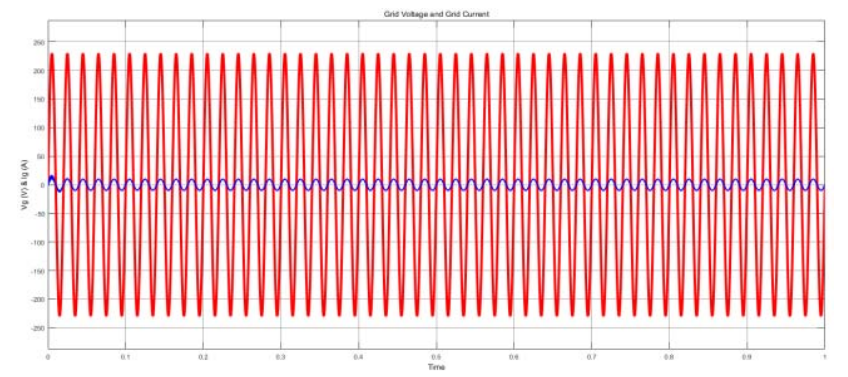

Fig. 12. Input Supply Voltage and current waveforms
The IEEE standard for THD ranges from $0.5 \%$ to $5 \%$. The proposed converter [18], projected that THD is less but after simulating the same circuit it is having current THD of $4.90 \%$ which is reasonably under the range but still on the higher scale of THD and the mentioned modes were giving the wide output voltage range in boost mode but buck mode is not conducive. The ZVS interleaved AC-DC converter proposed in [13] is having a THD closer to $15.2 \%$ which is not acceptable according to the standards. The projected converters in [14]-[16] shows a THD of $1.90 \%$ and $6 \%$ at rated current. The THD of input supply current is $2.80 \%$ in [19] proposed for PFC. From the simulated results of this converter are shown in the Fig. 10 - 13. These results clearly show us that distortion present in the input waveform is removed and the expected output remains the same and there is no compromise of getting the output range of this modified model. The output voltage and controllability of the output voltage remains the same as compared to the first proposed model. But this modified model removed the distortion and improved the THD.

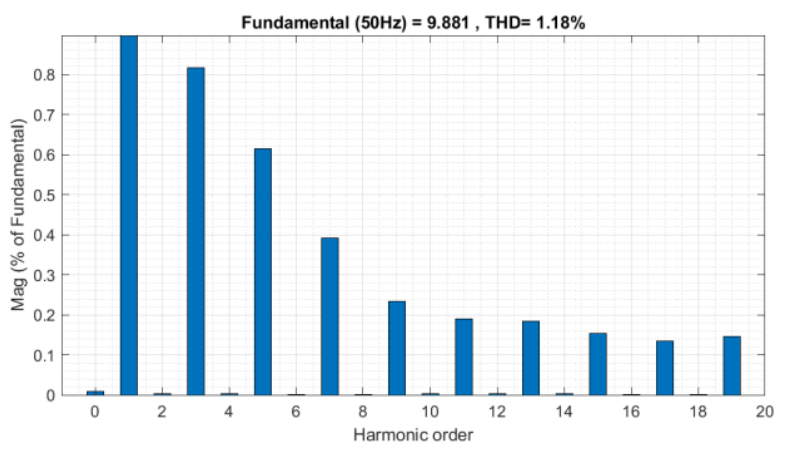

Fig. 13. THD of grid input current

As we can see from the above figure 13 that the THD for the proposed model is $1.18 \%$ which is a significant improvement compared to the original THD values of the above listed papers. Hence the power factor and power quality are better in this model while achieving the desired voltage required by the battery.

\section{Conclusions}

The proposed PFC converter operates with wide output voltages for universal input voltages. It produces a wide DC link voltage with smooth input current, even if the converter runs above and below the peak of the input voltage or rectified output voltage. By using interleaved combination at the input provides reduced ripple content in the input current. Over conventional converters, the proposed topology has a current THD of $1.18 \%$, lower ripple in input current, lower output voltage ripple, stress on switches are less, prompter transient response, superior reliability, and substantially lower electromagnetic interference. In addition, because the input current is split into two routes in the proposed topology, ohmic loss and inductor ac loss are significantly decreased, and the system ultimately accomplishes much greater 
efficiency. The IBBC PFC converter improves the charging power quality and efficiency which ultimately reduces the charging rates of an EV.The above features makes it possible for the proposed converter to be used in G2V technology related applications.

\section{References}

[1] B. M. Conlon, "How Much Capability Do Electric Vehicles Need to Meet Customer Demands? [Viewpoint]," in IEEE Electrification Magazine, vol. 5, no. 1, pp. 4-73, (March 2017).

[2] Yilmaz M, Krein PT, "Review of battery charger topologies, charging power levels, and infrastructure for plug-in electric and hybrid vehicles" IEEE Trans., on Power Electron. vol. 28, pp.2151-2169, (May 2013).

[3] Kondreddy Sreekanth Reddy, Sreenivasappa B. V, "Review on Power Converters for Electric Vehicles", Journal of Xi'an University of Architecture \& Technology, vol.7, pp.82-105, (Aug 2020).

[4] Ivan Subotic, Student Member, "A Review of Single-Phase On-BoardIntegrated Battery Charging Topologies for Electric Vehicles" IEEEWorkshop on Electrical Machines Design, Control and Diagnosis(WEMDCD) March 26-27, (2015)Torino, Italy.

[5] Siddhant Kumar, Adil Usman, “A Review of Power Converter topoplogies for battery charging applications in plug in Hybrid Electric Vehicle" IEEE industry Applications Society Annual meeting(IAS) conference, (Sept.2018).

[6] L. Huber, J. Yungtaek, and M. M. Jovanovic, "Performance evaluation of bridgeless PFC boost rectifiers," IEEE Trans. Power Electron., vol. 23, pp. 1381-1390, (May 2008).

[7] F. Musavi, W. Eberle, and W. G. Dunford, "A highperformance single-phase bridgeless interleaved PFC converter for plug-in hybrid electric vehicle battery chargers," IEEE Trans. Ind. Appl., vol. 47, no. 4, pp. 1833-1843, (Jul./Aug. 2011).

[8] Mahmoud Nassary, Ohamed Orabi, Maged Ghonema, "Discussion of single-stage Isolated unidirectional charger for Electric Vehicle" 2018 IEEE $4^{\text {th }}$ Southern Power Electronics Conference (SPEC).( December 2018)

[9] V. R. K. Kanamarlapudi, B. Wang, P. L. So and Z. Wang, "Analysis, Design, and Implementation of an APWM ZVZCS Full-Bridge DC- DC Converter for Battery Charging in Electric Vehicles," in IEEE Trans. on Power Electron., vol. 32, no. 8, pp. 61456160.( Aug. 2017).

[10] K. Sreekanth Reddy, Sreenivasappa B. Veeranna, "Design Parameters of Electric Vehicle", International Conference on Power Electronics \& IoT Applications in Renewable Energy and its Control (PARC), IEEE, (Feb 2020).

[11] Aman Jha, Bhim Singh, A bridgeless boost PFC converter fed LED driver for high power factor and low THD, IEEMA Engineer Infinite Conference (eTechNxT) (Jun.2018).

[12] Pratap Ranjan Mohanty; Anup Kumar Panda; Dhiman Das, "An active PFC boost converter topology for power factor correction", Annual IEEE India Conference (INDICON) (Dec. 2015).

[13] Sagar Sandeepan Indalkar;Anupa Sabnis, "An OFF Board Electric Vehicle Charger Based On ZVS Interleaved AC-DC Boost PFC Converter", 8th International Conference on Power Systems (ICPS), (Dec. 2019).

[14] J. Chen, D. Maksimovic, and R. W. Erickson, "Analysis and design of a low-stress buck-boost converter in universal-input pfc applications," IEEE Transactions on Power Electronics, vol. 21, no. 2, pp. 320-329,(March 2006).

[15] Kondreddy Sreekanth Reddy, Dr. Sreenivasappa B. Veeranna, "Single Phase Multifunctional Integrated Converter for Electric Vehcles", Indonesian Journal of Electrical Engineering and Computer Science (2021)(Accepted)

[16] Guirguis Z. Abdelmessih1, J. Marcos Alonso1, Marco A. Dalla Costa, Yu-Jen Chen, Wen-Tien Tsai," Fully Integrated Buck and Boost Converter as a High-Efficient High-Power-Density Off-Line LED Driver", 8th International Conference on Power Systems (ICPS), (Dec. 2019).

[17] X. S. Praneeth AV, Williamson S S, “ A wide input and output voltage range battery charger using buckboost power factor correction converter" IEEE Applied Power Electronics Conference and Exposition (APEC), (Mar 2019).

[18] Mihaela-Codruta Ancuti, Marcus SvobodA, Sorin Musuroi, Alexandru Hedes, Nicola-Valeriu Olarescu, Martin Wienmann, "Boost Interleaved PFC versus Bridgeless Boost Interleaved PFC Converter Performance/Efficiency Analysis", International Conference on Applied and Theoretical Electricity (ICATE), (Dec. 2014).

[19] J. Marcos Alonso, Juan Viña, David Gacio Vaquero, Gilberto Martínez, and René Osorio, "Analysis and Design of the Integrated Double Buck-Boost Converter as a High-Power-Factor Driver for Power-LED Lamps", IEEE Trans. On Industrial Electron., vol. 59, no. 4, (April 2012).

[20] Ashna Joseph and Jebin Francis, "Design and Simulation of Two Phase Interleaved Buck Converter" International Journal of Advanced Research in Electrical, Electronics and Instrumentation Engineering, Vol. 4, Special Issue 1, (March 2015). 